A
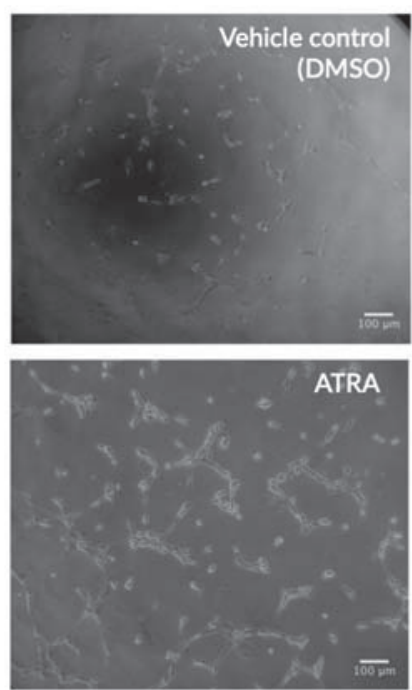

B

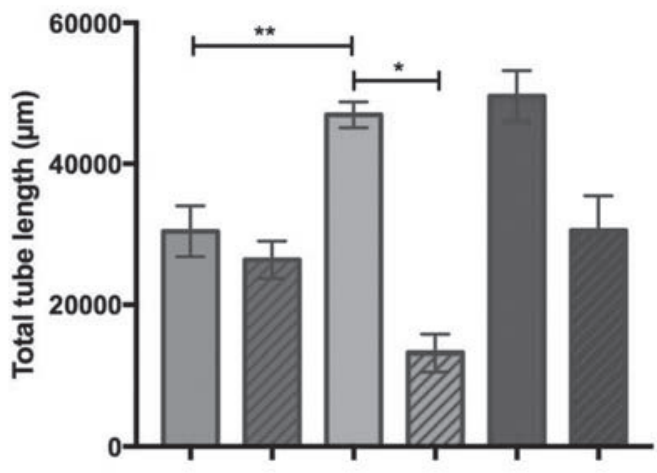

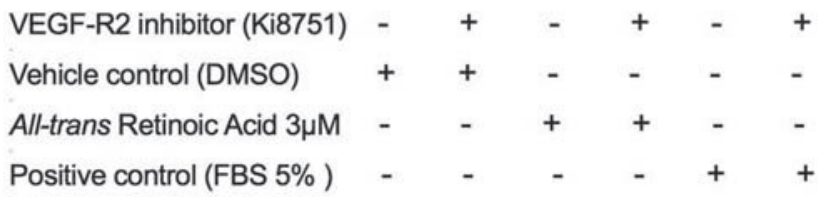

Abstract P45 Figure 1 ATRA induces pulmonary microvascular angiogenesis through VECF-Receptor 2. HPMECs were seeded onto matrigel and tube formation assessed after 4 hours. Representative images shown, original magnification x10 (A). ATRA $3 \mu \mathrm{M}$ significantly increased total tube formation when compared to vehicle control (DMSO 0.03\%) and this effect was abolished by the selective VEGF-Receptor 2 inhibitor Ki8751 (Tocris) (B). (Results presented as Mean $\pm S E M,{ }^{*} p<0.05$ Mann-Whitney test, $n=6$ (2 independent experiments with 3 replicates per condition).

Retinoic acid (RA) is essential for correct mammalian lung development and remarkably can induce alveolar regeneration in rodent models of COPD. However, clinical trials of RA and RA-Receptor gamma agonists in patients with COPD were disappointing. The reasons for the lack of effect in COPD patients are unknown but there is a clear need for a greater understanding of the underlying biology driving RA-induced lung regeneration. To investigate this, I studied the role of RA in human alveolar repair using isolated human pulmonary microvascular endothelial cells (HPMEC), alveolar epithelial cells (A549) and developed a novel human alveolar model using Precision-Cut Lung Slices (PCLS). In HPMECs, All-trans RA (ATRA) induced angiogenesis in a dose dependent manner $(\mathrm{p} \leq 0.01, \mathrm{n}=5)$. Pharmacological inhibition of VEGF receptor-2 with the selective inhibitor Ki8751 (Tocris) abolished this effect $(\mathrm{p} \leq 0.05, \mathrm{n}=6)$ (figure 1$)$. A proteome profiler array of PCLS, demonstrates an increase in pro-angiogenic proteins in the ATRA group, including CXCL16, IGFBP-3, PIGF, VEGFA, HB-EGF and MCP-1. In addition, ATRA treatment of PCLS generated from histologically normal human lung lead to increased endothelial (PECAM-1) and alveolar type 2 (ProSPC) cell markers $(n=3)$. Further investigation revealed that in wound-healing (scratch) assays of confluent cell monolayers, ATRA had no direct effect on the rate of wound healing in alveolar epithelial cells (A549) but significantly increased healing in HPMECs $(\mathrm{p} \leq 0.01, \mathrm{n}=3)$. Moreover, siRNA knockdown of VEGF-R2 inhibited ATRA-induced wound-healing in HPMECs. Conditioned media from ATRA-treated HPMEC increased wound healing in A549 cells suggesting that the effects of RA on alveolar epithelial repair are mediated indirectly via the vascular network. HPMEC secreted HB-EGF with ATRA stimulation and HB-EGF treatment significantly increased A549 wound healing $(n=2)$ suggesting it may act as a paracrine endothelial-epithelial regulator. My work demonstrates that RA has biological activity in human lung with direct effects on human lung microvasculature including cell migration, angiogenesis, and regulation of proteins likely to be important in alveolar repair. Together my data significantly advances our understanding of the mechanisms of RA induced repair in human lung tissue.

\section{P46 CIGARETTE SMOKE- AND HYPOXIA-INDUCED IMBALANCED VASOACTIVE GENE EXPRESSION IN HUMAN PULMONARY ARTERY ENDOTHELIAL AND SMOOTH MUSCLE CELLS}

A Alqarni, O Brand, A Pasini, M Alshehri, L Pang. Division of Respiratory Medicine, School of Medicine, University of Nottingham (City Hospital), Nottingham, UK

\subsection{6/thoraxjnl-2017-210983.188}

Background Pulmonary Hypertension (PH) is a common and serious complication of Chronic Obstructive Pulmonary Disease (COPD) associated with increased mortality and morbidity and characterised by Pulmonary Artery Smooth Muscle Cell (PASMC) hyperproliferation and vascular remodelling. Studies suggest that chronic hypoxia and Cigarette Smoke (CS) can cause aberrant PASMC proliferation and vascular remodelling, however, how cigarette smoke and hypoxia contribute to pulmonary artery wall thickening and $\mathrm{PH}$ in COPD is not fully understood. We hypothesise that hypoxia and CS can induce an imbalance between excessive vasoconstrictors and deficient vasodilators, which then contribute to aberrant PASMC proliferation in COPD-associated $\mathrm{PH}$ and can be a target for therapeutic intervention.

Method To prove the hypothesis, confluent Human Pulmonary Artery Smooth Muscle Cells (hPASMCs) and Human Pulmonary Artery Endothelial Cells (hPAECs) were treated with different concentrations of Cigarette Smoke Extract (CSE) (1\%, $2.5 \%$, and $5 \%)$ under normoxic $\left(21 \% \mathrm{O}_{2}\right)$ or hypoxic $(1 \%$ $\mathrm{O}_{2}$ ) condition for 72 hour. The protein and mRNA expression of Prostacyclin Synthase (PGIS), Cyclooxygenase-2 (COX-2), Endothelial Nitric Oxide Synthase (eNOS), Thromboxane A Synthase (TXAS), and Endothelin 1(ET-1) was analysed by Western blotting and real-time RT-PCR, respectively.

Results The expression of vasodilator genes eNOS and PGIS was noticeably downregulated in both hPASMCs and hPAECs, 
whereas TXAS and COX-2 expression was markedly induced by CSE and hypoxia, either individually or in combination in both hPASMCs and hPAECs. ET-1 expression was increased by CSE and hypoxia in hPAECs. Interestingly, ET-1 was upregulated by hypoxia, but reduced by CSE, with a net increase when both were combined in hPASMCs.

Conclusion These findings support our hypothesis that CS and hypoxia can cause an imbalance between excessive vasoconstrictors and deficient vasodilators in hPASMC and hPAECs. This imbalance may eventually lead to aberrant PASMC proliferation and vascular remodelling in COPD-associated PH. Further experiments are being conducted to confirm this by analysing vasoactive gene expression and mediator release in both hPASMCs and hPAECs. Our findings also pave the way for further studies on cellular functions and intervention drug effects.

\section{P47 HYPERCAPNIA IMPAIRS THE ABILITY OF MESENCHYMAL STEM CELLS TO PROMOTE DISTAL LUNG EPITHELIAL WOUND REPAIR IN ARDS}

NF Fergie, DF McAuley, CM O'Kane, AD Krasnodembskaya. Queen's University Belfast, Belfast, UK

\subsection{6/thoraxjnl-2017-210983.189}

Background Alveolar epithelial cell death and denudation of the basement membrane are hallmarks in the pathophysiology of Acute Respiratory Distress Syndrome (ARDS). Successful recovery requires basement membrane re-epithelialisation. While no pharmacological therapy exists for ARDS to date, Mesenchymal Stem Cells (MSCs) demonstrate promising therapeutic potential and are being tested in early-phase clinical trials. Heterogeneity of patients with ARDS is an obstacle for further development of an MSC-based therapy. While 20\% of patients with ARDS develop hypercapnia (high $\mathrm{CO}_{2}$ ) as a result of lung protective ventilation, the efficacy of MSCs has never been studied in this setting. We have previously found that transfer of functional mitochondria to surrounding cells is an important mechanism of the MSC therapeutic effect. The aims of this study therefore were to investigate the effect of MSCs on repair of the distal lung epithelium in normocapnia and hypercapnia in an in vitro model of ARDS, and to assess the role of mitochondrial transfer in mediating the MSC effect.

Methods Primary, human small airway epithelial cell (SAEC) monolayers were wounded in an in vitro scratch assay, stimulated with cytomix (IFN-gamma, I L-1 beta, TNF-alpha), and co-cultured with MSCs in normocapnia $\left(5 \% \mathrm{CO}_{2}\right)$ or hypercapnia $\left(15 \% \mathrm{CO}_{2}\right)$. Percentage wound closure was measured at 24 hour. SAEC proliferation was assessed by Ki67 staining. Mitochondrial transfer from MSCs to SAECs was assessed by flow cytometry using MitoTracker Green dye. MSC mitochondrial membrane potential was analysed by flow cytometry using JC-1. ATP production was measured by luminescent assay.

Results Epithelial wound closure was impaired by hypercapnia. MSCs promoted epithelial wound closure in the inflammatory setting in normocapnia via enhanced migration. This reparative capacity was lost in hypercapnia. Mitochondrial transfer from
MSCs to SAECs was observed to a similar extent in normocapnia and hypercapnia. However, hypercapnia attenuated mitochondrial membrane potential and ATP production in MSCs.

Conclusion While they promote epithelial wound repair in an inflammatory environment in normocapnia, MSCs lose this ability in hypercapnia. This suggests that their therapeutic efficacy may be lost in such an environment. An inhibitory effect of hypercapnia on MSC mitochondrial function may be at least partially responsible for this effect.

\section{P48 THE EFFECTS OF TGF-B AND IL-33 ON THE PRO- FIBROTIC ACTIVITY OF PRIMARY HUMAN LUNG FIBROBLASTS DURING THE DEVELOPMENT OF IPF}

${ }^{1} \mathrm{KE}$ Stephenson, ${ }^{2} \mathrm{CL}$ Overed-Sayer, ${ }^{1} \mathrm{AE}$ John, ${ }^{2} \mathrm{ES}$ Cohen, ${ }^{1} \mathrm{RG}$ Jenkins. ${ }^{1}$ University of Nottingham, Nottingham, UK; ${ }^{2}$ Medlmmune Ltd, Cambridge, UK

\subsection{6/thoraxjnl-2017-210983.190}

Introduction Idiopathic pulmonary fibrosis (IPF) is a debilitating interstitial lung disease with a poor prognosis and limited treatment options. It is a chronic, progressive, condition characterised by the excessive deposition of extracellular matrix by fibroblasts. Transforming growth factor- $\beta$ (TGF- $\beta$ ) is central to this process and is regarded as a key pro-fibrotic mediator in IPF. Recently, emerging evidence suggests that the cytokine interleukin-33 (IL-33) may also be important in the development of IPF. However, the cellular and molecular mechanisms by which IL-33 promotes fibrosis are unknown. In particular, whether TGF- $\beta$ and IL-33 have independent pro-fibrotic effects on fibroblasts remains unclear.

Methods Primary human lung fibroblasts (HLFs) from non-IPF and IPF patients were treated with either $2 \mathrm{ng} / \mathrm{ml}$ TGF- $\beta$ or $10 \mathrm{ng} / \mathrm{ml} \mathrm{IL-33.} \mathrm{The} \mathrm{levels} \mathrm{of} \mathrm{IL6} \mathrm{and} \mathrm{IL8} \mathrm{mRNA} \mathrm{were} \mathrm{used}$ as readouts of IL-33-mediated NF- $\kappa \beta$ activation whilst ACTA2 and COL1A1 mRNA were used as readouts of fibrosis. mRNA levels were measured by real-time quantitative reverse transcription polymerase chain reaction (qRT-PCR). Finally, the levels of IL-33, and its receptor ST2, were measured by qRTPCR to assess mRNA expression and western blotting to measure protein.

Results Stimulation with TGF- $\beta$ for 8 hours induced statistically significant increases in IL33 gene expression by both non-IPF and IPF HLFs. Moreover, TGF- $\beta$ stimulation of HLFs from patients with and without IPF induced IL-33 protein as assessed by immunoblotting. To assess the functional consequence of TGF-b induced IL-33 from fibroblasts, the effect of recombinant IL-33 on HLFs was determined. Increasing concentrations of IL-33 failed to stimulate IL6, IL8, ACTA2 and COL1A1 gene expression by either IPF or non-IPF fibroblasts. To understand the lack of IL-33 responsiveness in HLFs, ST2 levels were measured and neither ST2 mRNA or protein were detectable in IPF, or non-IPF, HLFs.

Conclusion TGF- $\beta$ increases IL-33 production by HLFs, however fibroblast-derived IL-33 does not act directly on fibrotic fibroblasts as they fail to express the receptor required for IL33 responsiveness. Therefore, the pro-fibrotic effects of IL-33 are likely mediated via alternative cell types. 